\title{
PHOTOMETRIC OBSERVATIONS AND REDUCTIONS OF LIGHTCURVES OF ASTEROIDS
}

\author{
RONALD C. TAYLOR \\ University of Arizona
}

The brightness-time variation (the lightcurve) of an asteroid is observed to obtain the rate of rotation, some indication of the shape, and the orientation of the rotation axis in space. The brightness-phase relations are observed for the study of surface texture.

This paper deals specifically with observing routines and reductions, including discussions of lightcurves, rotation periods, absolute magnitudes, phase coefficients, opposition effects, and pole determinations. This paper supplements the review chapter by Gehrels (1970). Only photoelectric techniques are considered because the visual and photographic ones are, nearly without exception, not precise enough.

\section{OBSERVING ROUTINE}

Photoelectric observations of asteroids were made as early as 1935 by Calder (1935). Calder already observed comparison stars, chosen for their proximity to the asteroid and for similarity in color and magnitude. The comparison star observations allow correction for photometric changes in the quality of the night and to remove extinction effects from the lightcurve. (This, of course, assumes that the comparison star does not vary during the night.) A value for scatter of the comparison readings can be computed as an indication of the quality of the night. Because it is impossible-with a single detector-to observe the comparison star and the asteroid at the same time, interpolation of comparison star readings is necessary. The deviation of the comparison star readings from a smooth secant $Z$ curve is, at good sites as the McDonald and Kitt Peak Observatories, on the order of 0.01 mag; such effects remain uncorrected if no comparison star is observed (for instance, by Miner and Young, 1969, 1971). With careful comparison star corrections, the precision of the lightcurves generally is \pm 0.003 mag (for the mean of three measurements). With a two-detector photometer this may be improved to \pm 0.001 mag. (See Gehrels, 1970, fig. 4.)

With slight variations, the generally adopted observing routine is as follows: At the beginning and end of each run a red and a blue standard star 
are observed for $B, V$, and $U$. The order of the lightcurve observations is $A A S A A, C C, A A S A A, C C$, etc., where $A$ represents the asteroid, $C$ the comparison star, and $S$ the sky readings. Automatic lightcurve reductions during the night of observing have not as yet been made. As a step toward that goal we have a preliminary automated lightcurve reduction program.

Standard stars (not to be confused with the comparison star) are observed to determine the magnitude of the asteroid; generally. the UBV system of Johnson and Morgan (Johnson, 1963) is used. If one has a fast-moving telescope, the transfers can be performed during the lightcurve run rather than at the beginning and end of the night. Figure 1 illustrates the transfer routine.

In certain circumstances, one may find it desirable to forsake standard star observations during an asteroid run. In those cases, the comparison stars-and more than one should be observed because of possible variations-can be tied in to standard stars on subsequent evenings.

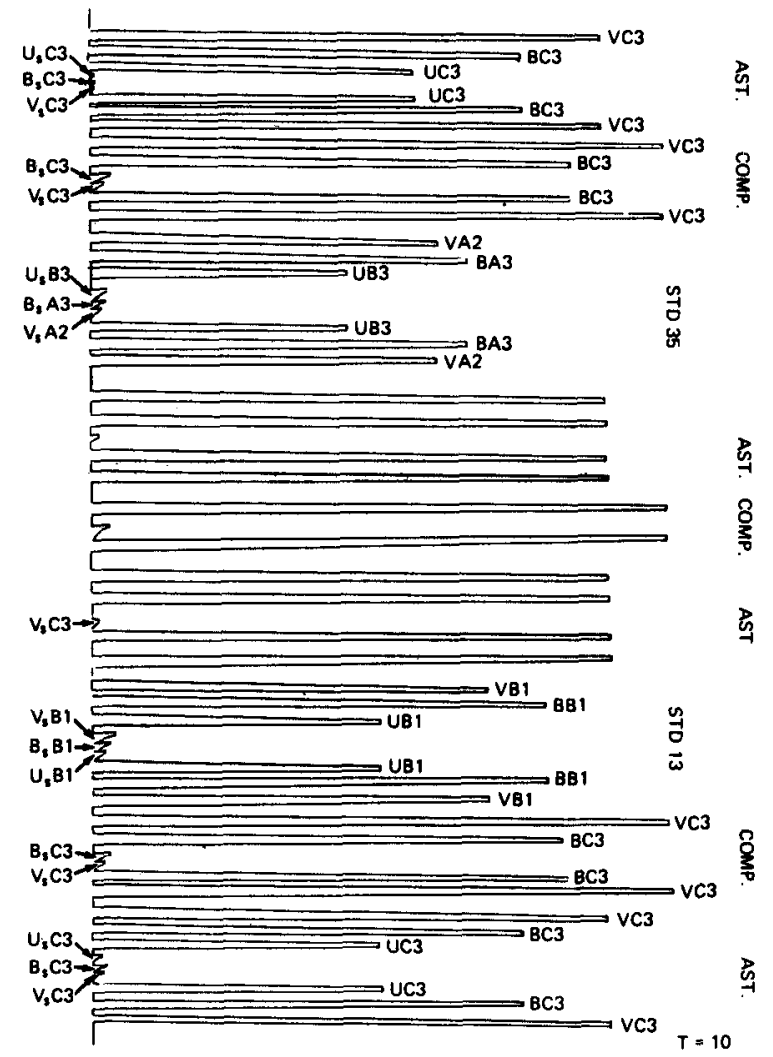

Figure 1.-A reproduction of $U B V$ transfer. $V, B$, and $U$ refer to the filters; the symbols $A 1$ to $C 3$ indicate gain setting; the subscript $s$ shows sky readings; and $T$ is the integration time. 
As for color variations, Groeneveld and Kuiper (1954a) observed in $V$ and $B$, but the color variations were only about $0.004 \mathrm{mag}$; therefore, they discontinued the procedure. Thirty color observations near maxima and minima were done in one evening on 624 Hektor. Little color differences were found, which supports the contention that the large amplitudes (as high as 1.1 mag) of the lightcurves were caused mostly by shape, rather than by a spotty surface (Dunlap and Gehrels, 1969). For Vesta, a slight variation of the $U-V$ color over the surface has been found (Gehrels, 1967a). To make a thorough study of color differences over the surface of an asteroid, a multichannel photometer may be an ideal instrument for the observations.

\section{LIGHTCURVE REDUCTIONS AND SYNODIC PERIODS}

Most authors have adapted the procedure of plotting each point on the lightcurve as the average of a set of values of the asteroid reading minus the comparison star reading with skies subtracted out, and an extinction correction applied. Generally, open circles have been used to represent uncertain values and, at times, authors have reproduced points to complete a lightcurve. Investigators have varied in taking the mean of from two to four asteroid readings per lightcurve point. In 1967, Gehrels (1967a) suggested that for bright objects "... it will be better always to plot the mean of two integrations rather than four, in order to show fine details of the shape of the lightcurve."

It appears that most lightcurves have been plotted using, as an ordinate scale, an arbitrary delta magnitude system between the comparison star and the asteroid. Occasionally authors have used a scale based on the $V$ magnitude of the asteroid. Generally lightcurves appear as a plot of points as indicated in figure 2. For clarity, some authors have joined mean lightcurve points with a curve, especially if the lightcurve is not well defined.

Because of the rapid motion of Icarus, corrections for phase and distance, as well as differential extinction, were needed to adjust each lightcurve. I reduced the raw data supplied by Miner and Young (Gehrels et al., 1970) for June 19 and 20,1968, by the general methods described above. Miner and Young (1969) averaged 3 to 12 data points within a 2.7 min segment, determined a

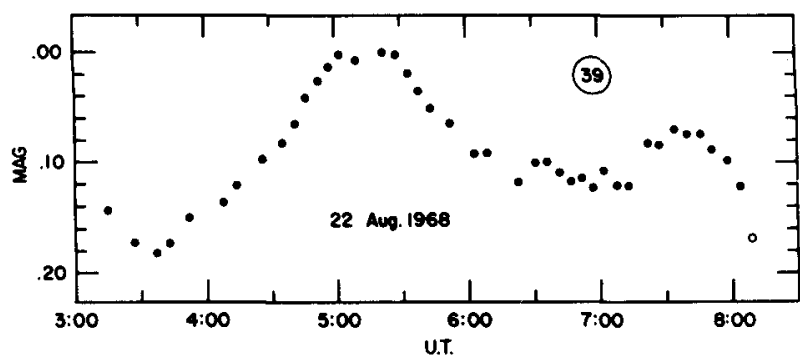

Figure 2.-A typical lightcurve. 
mean lightcurve with error bars averaging $\pm 0.015 \mathrm{mag}$, and smoothed the curve by successively fitting 5 consecutive points to a third-order polynomial. It is of interest to see how the two techniques create different lightcurves. (See figs. 3 and 4.)

Several authors have created mean lightcurves from various observations of the same asteroid. Generally, corrections of some fashion have been applied. Three such techniques are based on phase distance, rotation period, or comparison star magnitude differences (Chang and Chang, 1962; Groeneveld and Kuiper, 1954a; van Houten-Groeneveld and van Houten, 1958). With 20 Massalia phase changes caused amplitude variations, therefore a mean lightcurve was constructed from only two intermediate evenings (Gehrels, 1956). In analyzing 18 lightcurves of 6 Hebe over 3 oppositions, I have concluded that mean lightcurves can be made only when the observations are grouped by opposition and similar phase angles (Taylor and Gehrels, 1972). Figure 5 illustrates three mean lightcurves of Hebe. The various causes for the mean lightcurves to look different at various oppositions are discussed by Dunlap. ${ }^{1}$

Synodic periods have generally been determined by an inspection of the lightcurves. On occasion one must inspect the mean lightcurve, especially if the period is long and each lightcurve is short (Chang and Chang, 1962). The mean lightcurve was used with Massalia. The period was determined by dividing the number of cycles into the time intervals between maxima of consecutive lightcurves and of each lightcurve with the mean lightcurve (Gehrels, 1956).
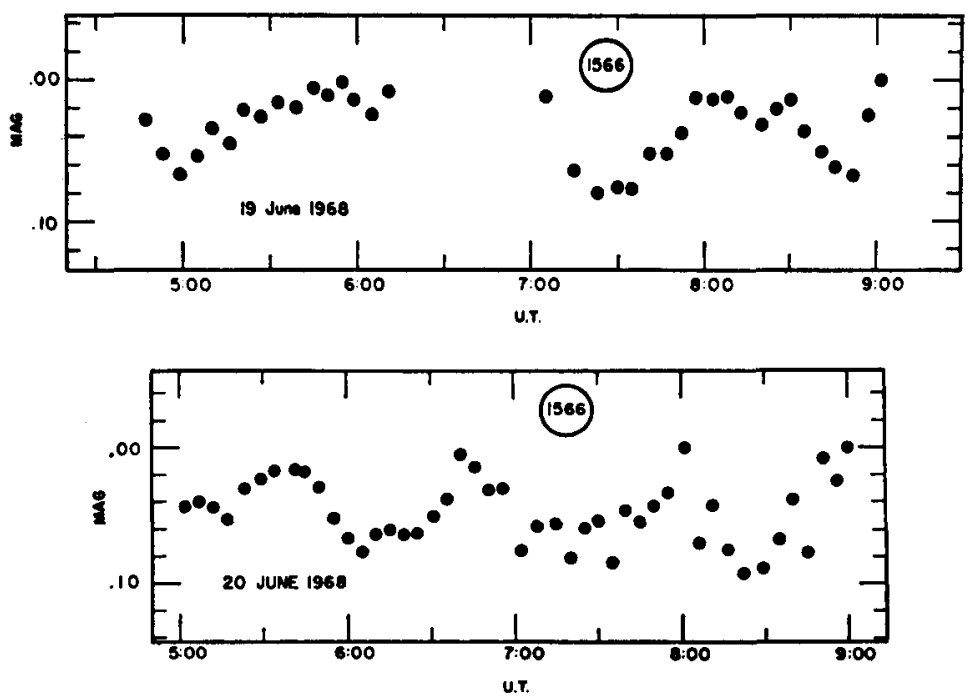

Figure 3.-Icarus data of Miner and Young reduced by standard method.

${ }^{1}$ See p. 147. 


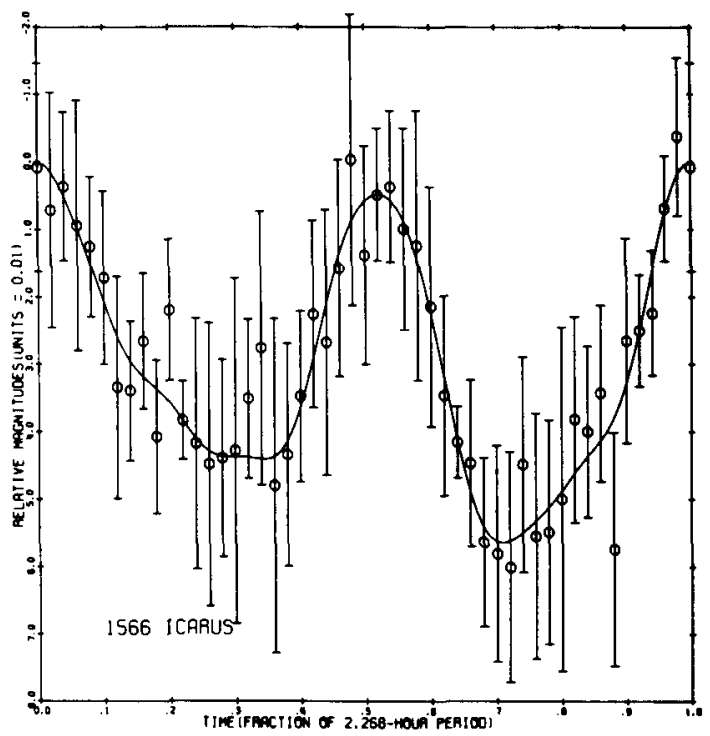

Figure 4.-Icarus data of Miner and Young reduced with their polynomial method.

The period can also be determined with the method of Hertzsprung (1928, $1941)$, which requires a least-squares routine on the ordered pair $(N, T)$, where $N$ is the number of cycles since the first epoch and $T$ is the time of each epoch in Julian days corrected for light time.

In fitting lightcurves of Vesta for a synodic period determination, it was assumed that the horizontal time axes are parallel. Because of phase changes, only time intervals from adjacent lightcurve epochs were used (Gehrels, 1967a). With Hebe, I found that in matching lightcurves one should not necessarily attempt to fit epochs of maximum or minimum light. Comparing these epochs can be misleading because there is evidence that maxima may

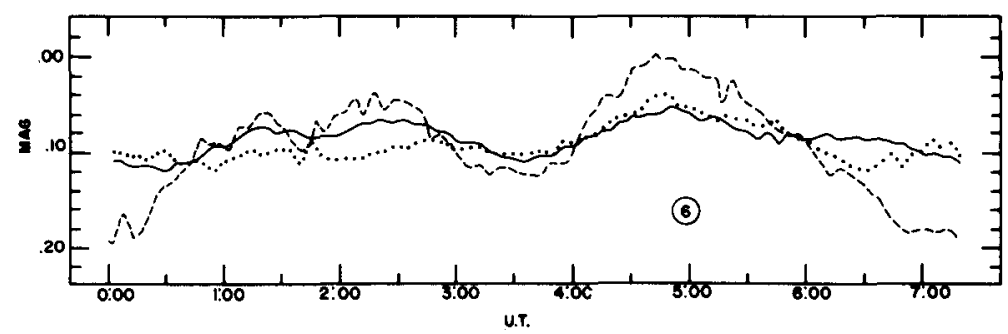

Figure 5.-Mean lightcurves of Hebe. Solid line, seven lightcurves of January and February 1959 at about $5^{\circ}$ phase; dotted line, four lightcurves of November and December 1958 and May 1959 at about $22^{\circ}$ phase; dashed line, three lightcurves of August 1968 at about $20^{\circ}$ phase. 
shift with reference to time; in fact, maxima or minima sometimes actually disappear (Taylor and Gehrels, 1972).

\section{ABSOLUTE MAGNITUDE, PHASE COEFFICIENT, AND OPPOSITION EFFECT}

Absolute magnitude $V(1,0)$ is defined as the $V$ magnitude at unit distance from the Sun and Earth at zero-degree phase angle. The phase coefficient refers to the magnitude change per degree of phase. The two values are directly related. Initially, phase coefficients can be determined from just two observations at different phase angles. For example, Groeneveld and Kuiper, with two observations of Eunomia, noted that after allowing for magnitude differences due to distance, there was still a discrepancy of $0.068 \mathrm{mag}$, which they deduced was due to a phase angle change of $1^{\circ} .6$. They therefore concluded that the phase coefficient was $0.042 \mathrm{mag} / \mathrm{deg}$ (Groeneveld and Kuiper, 1954a). The same technique has been used by others. Once phase coefficients have been adapted, a preliminary $V(1,0)$ can be determined as was done using Müller's (1897) average phase coefficients, with known distances and phase for 2 Pallas and 14 Irene (Groeneveld and Kuiper, 1954b). There are limitations: With 511 Davida it was found that for two lightcurves the phase angle changed $3^{\circ}$, the aspect about $90^{\circ}$, but the $V(1,0)$ values varied by 0.25 mag. Groeneveld and Kuiper (1954a) concluded that it was not entirely phase variation, and they warned that " . . the determination of the phase coefficient may contain an effect of area change, resulting from a change in aspect."

Hektor had similar type lightcurves; i.e., not sinusoidal and with large amplitude variations; and more consistent values of $V(1,0)$ were computed by using $V_{0}$ rather than mean $V$ (Dunlap and Gehrels, 1969). $V_{0}$ is the magnitude of the primary maximum of the lightcurve, whereas mean $V$ is the magnitude at a line on the lightcurve such that the areas enclosed above and below are equal. For Massalia, mean $V$ was used with the additional technique of analyzing the comparison stars in relation to the mean lightcurve to determine the magnitude of the asteroid $V_{\text {ast }}$ (Gehrels, 1956).

If several observations exist over a wide range of phase, $V_{\text {ast }}$, corrected for distance, can be plotted as a function of phase. Gehrels $(1967 \mathrm{~b})$ made a plea to define $V(1,0)$ as the extrapolated $V$ value at zero-degree phase. If one assumes a linear plot, then the slope is the phase coefficient. Twenty-two asteroids were observed and the conclusion drawn that for phase angles between $10^{\circ}$ and $20^{\circ}$ the average phase coefficient of the asteroids is $0.023 \mathrm{mag} / \mathrm{deg}$ (Gehrels, 1957).

Average $B-V$ and $U-B$ values have been plotted versus phase. Giclas (1951) and Haupt (1958) discovered a reddening with phase for various asteroids. No evidence of this phenomenon was found with Icarus (Gehrels et al., 1970). A reddening with phase was found for 110 Lydia (Taylor, Gehrels, and Silvester, 1971) and for 4 Vesta (Gehrels, 1967a).

Table I is an updated version of table III of Gehrels (1970); the absolute magnitude $B(1,0)$ and the mean opposition magnitude $B(a, 0)$ are taken from 


\begin{tabular}{|c|c|}
\hline 怤 & 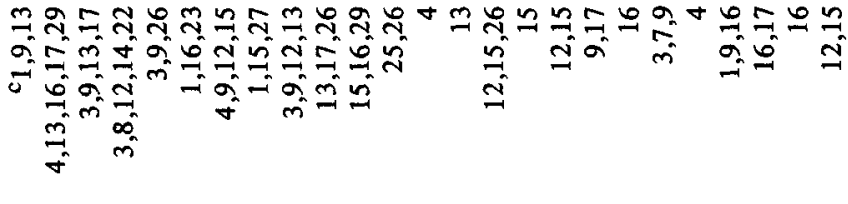 \\
\hline 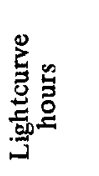 & 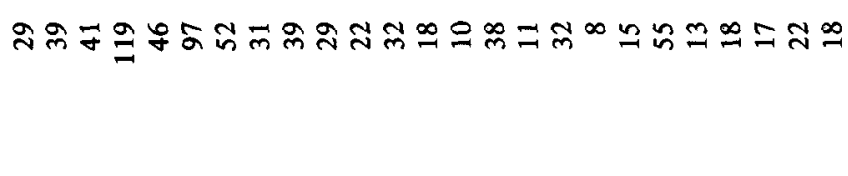 \\
\hline 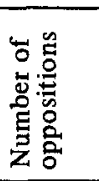 & $n+m b m+n n+n m-\pi n-n-7 m-m n-N$ \\
\hline 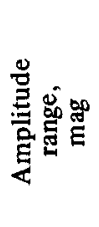 & 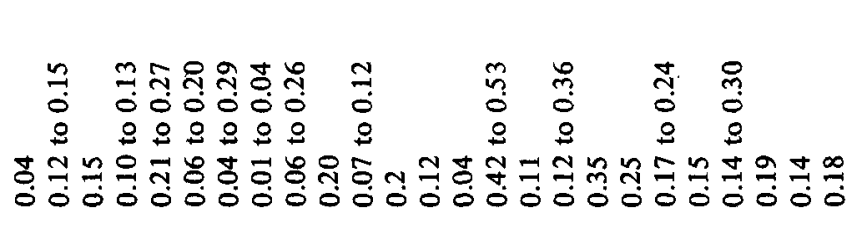 \\
\hline  & 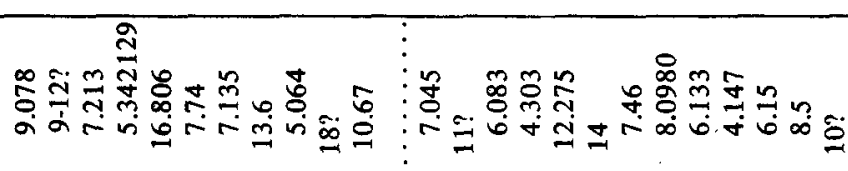 \\
\hline 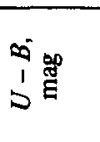 &  \\
\hline sig & 궁 \\
\hline $\begin{array}{l}\frac{2}{2} \\
0 \\
0\end{array}$ & 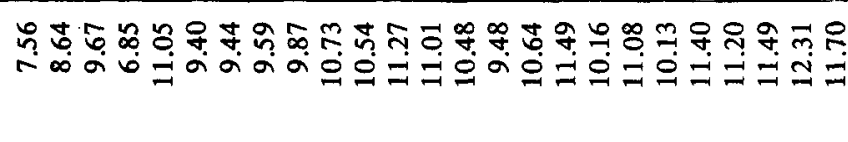 \\
\hline$\stackrel{0}{i}$ & 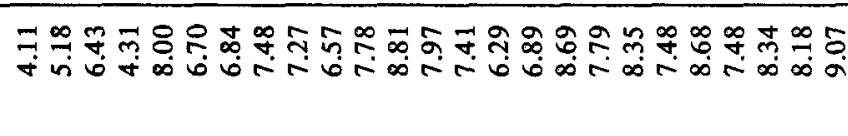 \\
\hline : & 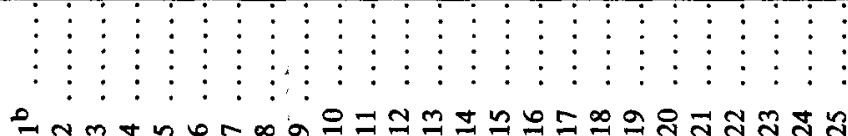 \\
\hline
\end{tabular}




\begin{tabular}{|c|c|}
\hline 总 & 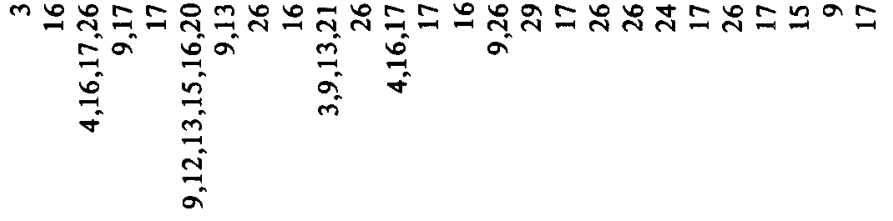 \\
\hline 胥 & 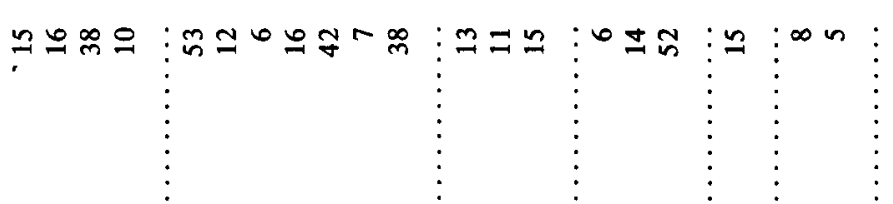 \\
\hline 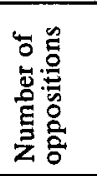 & 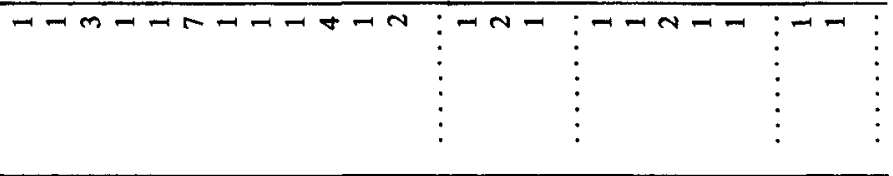 \\
\hline 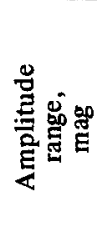 & 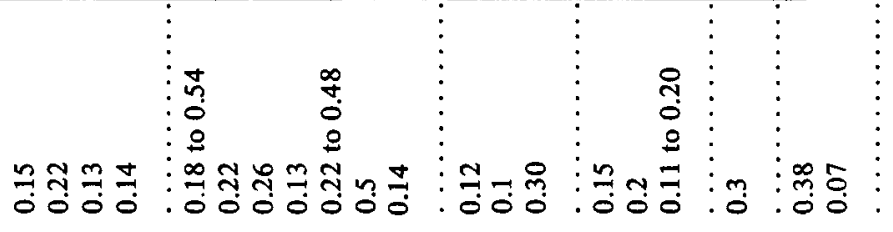 \\
\hline 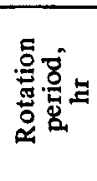 & 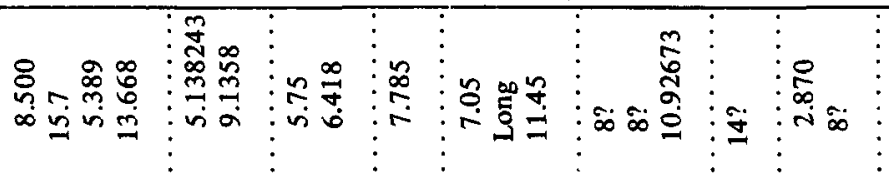 \\
\hline$\sum_{\substack{\infty \\
0}}^{\infty}$ & 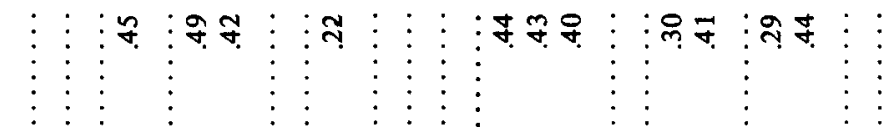 \\
\hline ing & 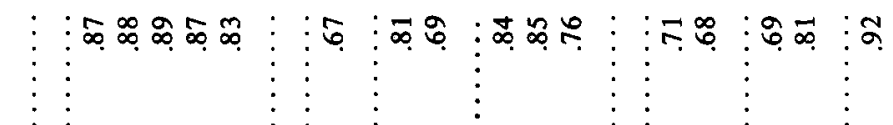 \\
\hline $\begin{array}{l}0 \\
0 \\
0\end{array}$ & 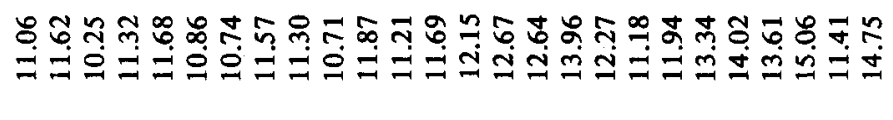 \\
\hline$\stackrel{0}{=}$ &  \\
\hline : & 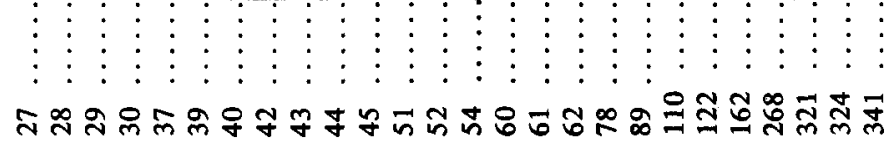 \\
\hline
\end{tabular}




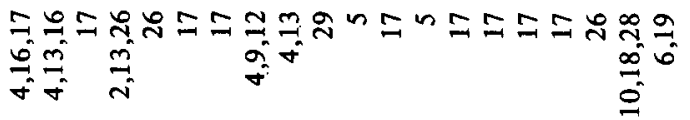

苂每

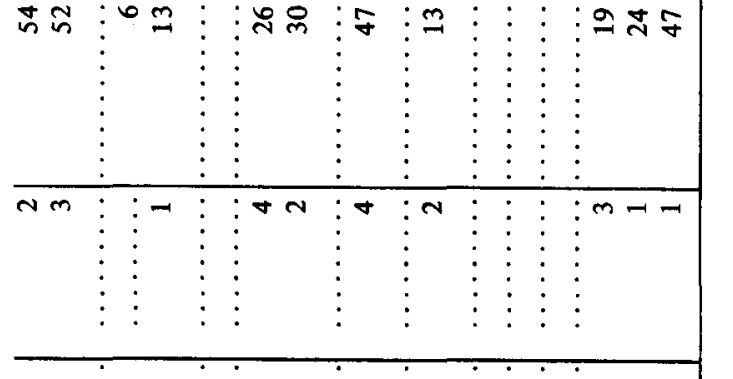

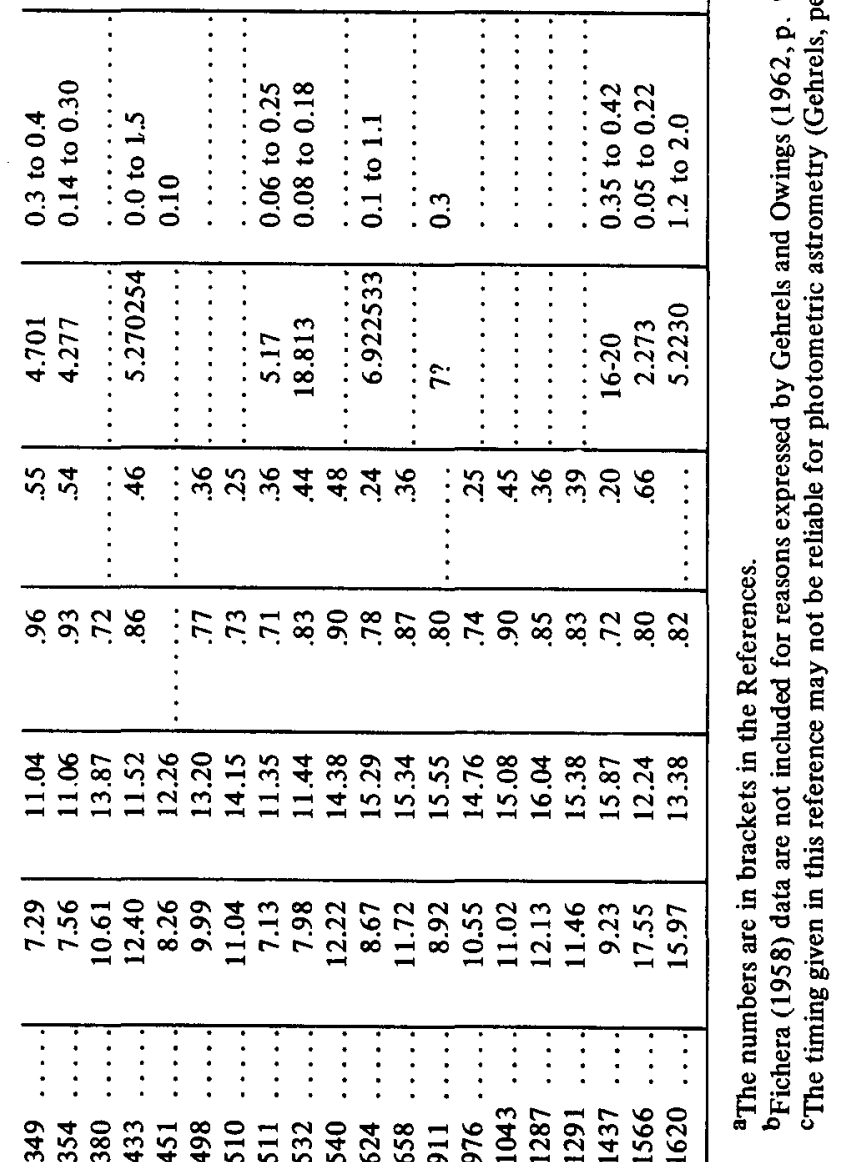


his table II. The $B-V$ and $U-B$ columns are the colors at $5^{\circ}$ phase; the period is synodic unless the sidereal period is known. Amplitude ranges and the number of oppositions observed are indicated. The hours column refers to the number of hours of good lightcurves obtained.

Massalia was observed for the expressed purpose of determining magnitudephase relations at small phase angles, and the opposition effect was discovered: a sharp increase in brightness from $7^{\circ}$ phase on toward $0^{\circ}$ phase (Gehrels, 1956). Figure 6 illustrates the phase relations along with the opposition effect as they appeared with Lydia (Taylor, Gehrels, and Silvester, 1971). With Lydia, when $B$ and $U$ were plotted as a function of phase, it appeared that the opposition effect was independent of wavelength. Also, the opposition effect for Massalia, Vesta, and Lydia appeared the same, as is illustrated in figure 7 (Taylor, Gehrels, and Silvester, 1971).

Certain asteroids should perhaps be reevaluated in view of our present knowledge of the opposition effect. Three examples are as follows:

(1) The absolute magnitude and phase coefficient of 9 Metis was determined using five observations, four of which were under $6^{\circ}$ phase (Groeneveld and Kuiper, 1954b).

(2) Observations of Iris near $23^{\circ}$ phase yielded consistent $V(1,0)$ values but a later run at $4^{\circ}$ phase was 0.2 mag brighter than expected (van Houten-Groeneveld and van Houten, 1958).

(3) $V(1,0)$ was found to be different by $0.01 \mathrm{mag}$ for 12 Victoria before and after opposition. For Victoria it was assumed that the opposition effect started at phase angles less than $5^{\circ}$. In the linear plot after opposition, three of four data points lie in the region of $5^{\circ}$

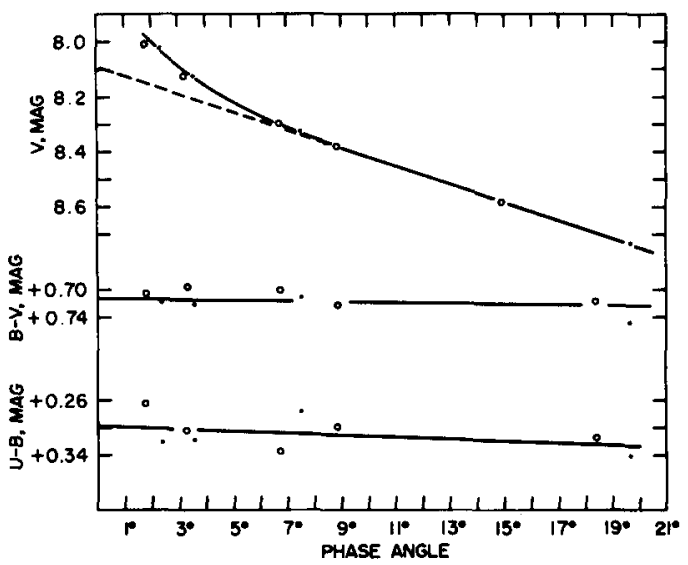

Figure 6.-Phase functions of Lydia. Ordinates: top curve, the observed magnitudes ( $V$ on the $U B V$ system) reduced to unit distances from the Sun and Earth; middle curve, the $B-V$ colors; bottom curve, the $U-B$ colors. Open circles are before opposition and filled circles are after opposition. 


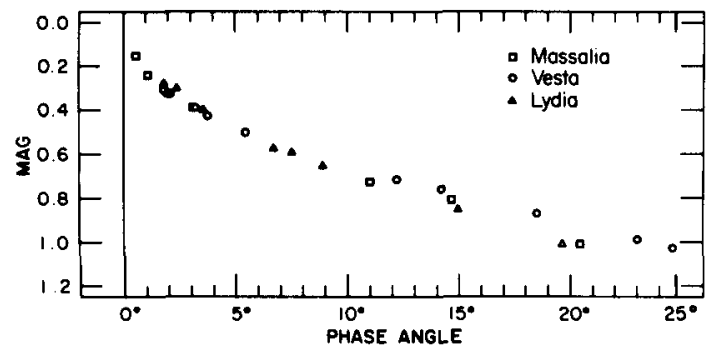

Figure 7.-Magnitude-phase relations of three asteroids.

to $6^{\circ}$ phase (Tempesti and Burchi, 1969). If the assumption is made that the opposition effect starts at $7^{\circ}$ to $8^{\circ}$ phase, I feel there may be a unique $V(1,0)$.

\section{SENSE OF ROTATION AND POLE DETERMINATIONS}

To determine the sense of rotation of Eunomia, Groeneveld and Kuiper (1954a) assumed the ecliptic latitude of the pole to be at $90^{\circ}$ and used the relation

$$
N P=\Delta t_{c} \pm \frac{\Delta L}{360} P
$$

where $N$ is the number of cycles, $P$ is the period, $\Delta t_{c}$ is time interval corrected for light time, $\Delta L$ is the difference in ecliptic longitudes, the plus sign is used for retrograde rotation, and the minus sign for direct rotation.

A retrograde solution gave smaller residuals in $P$ for the two intervals attempted. "Clearly ... some uncertainty may be introduced ... by the assumptions made concerning the position of the rotational axis" (Groeneveld and Kuiper, 1954a). The van Houtens with additional data and using the same method confirmed retrograde rotation; they present a concise and informative discussion of limitations in their "Concluding Remarks" (van HoutenGroeneveld and van Houten, 1958).

The sense of rotation of Vesta was determined by using many intervals of both increasing and decreasing longitudes, and by assuming that the latitude of the pole was high (Gehrels, 1967a).

Groeneveld and Kuiper (1954a) presented a pole determination method and claim 10 percent precision if optimum conditions are met: axis fixed in space and an observation at each of the stationary points of two successive oppositions.

With 39 Laetitia, a formula was developed to adjust the period because of the relative motions of Earth and the asteroid. The formula depends on 
knowing the pole orientation. An amplitude-aspect relation derived by Stobbe (1940) and Beyer (1953) for Eros, scaled down, was applied to determine an estimate of the pole. The investigators admittedly had limited precision (van Houten-Groeneveld and van Houten, 1958).

The poles of eight asteroids were calculated by combining two techniques: a sine relation between aspect and amplitudes, and a cosine relation between absolute magnitude changes with respect to aspect (Gehrels and Owings, 1962). It is not clearly established whether a sine relation is proper for those asteroids studied.

With his work on Vesta, Gehrels developed what is now known as "photometric astrometry." The method is basically the same as that used by Groeneveld and Kuiper for finding the sense of rotation of Eunomia. The main difference is that Gehrels did not restrict his analysis to a $90^{\circ}$ orientation of the pole. He considered the asteroid-centric longitude changes between observations for various pole possibilities. Those differences were applied as corrections to the number of cycles for each interval. By attempting different orientations, he sought minimum residuals from the mean sidereal period of each trial. His method is "... independent of any assumptions regarding the shape of the asteroid and the relationship between amplitude and the aspect." $\mathrm{He}$ also introduced a phase shift to correct for the displacement of the center of light on the apparent disk due to the effects of phase. Gehrels compared his data with earlier observations, over 20000 cycles, to improve the precision (Gehrels, 1967a); that part of the analysis will, however, have to be redone, as is planned for a future paper, because the additional cycle correction for each orbital revolution was omitted.

In the Hektor analysis (Dunlap and Gehrels, 1969), photometric astrometry was used, but it was difficult to determine the number of cycles. There were only a few observations over long intervals. As an aid, the relation $\Delta N= \pm N\left(P_{\text {sy n }}-P_{\text {sid }}\right) / P_{\text {sid }}$ was used, where $P_{\text {sy } n}$ is the synodic period and $P_{\text {sid }}$ is the sidereal period. Figure 8 shows how the apparent number of cycles $\Delta N$ is changed as a function of longitude for four different pole orientations. That figure assumes the asteroid is on the ecliptic, as was the case with Hektor.

Figure 8 could not be used for Icarus because the asteroid was not on the ecliptic. Figure 9 shows how the apparent number of cycles are affected if the asteroid is $20^{\circ}$ above the ecliptic. The entire photometric astrometry routine, including the problems of cycles, was computerized before the Icarus analysis. The concept of light centers was also introduced: the center of the projection of the illuminated part of the disk, as seen from Earth, assuming uniform reflectivity and a spherical shape. The light center is on the great circle through the subsolar and sub-Earth points. The purpose for light centers is basically the same as for Gehrels' phase shift (Gehrels et al., 1970).

In conclusion, it is clearly seen that additional work is needed to improve the quality and the extent of the sample in table $I$. I feel that high priority should be given to improving pole determinations. For this purpose, high 


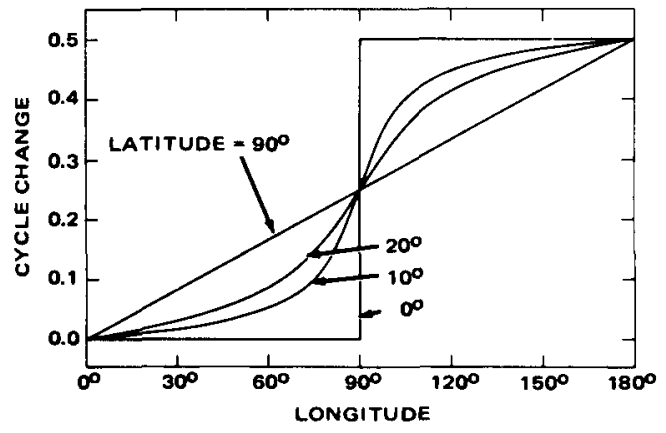

Figure 8.- The apparent change in cycle of asteroid rotation at various longitudes, plotted for four values of the latitude of the pole, $90^{\circ}$ longitude of pole; asteroid is on the ecliptic.

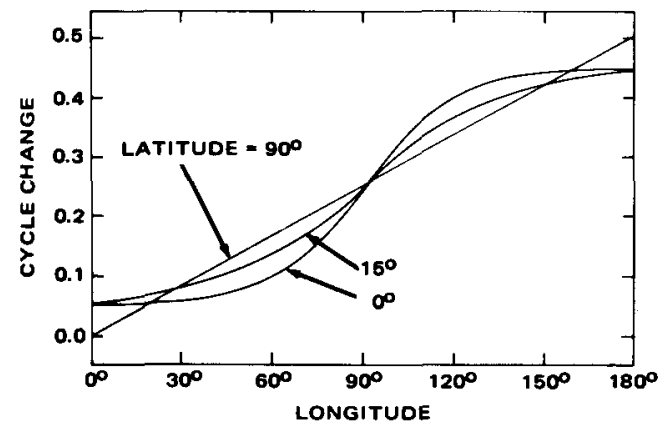

Figure 9.-Same as figure 8 except asteroid is $20^{\circ}$ above the ecliptic.

precision is needed in the timing of lightcurves to establish precise epochs of maximum or minimum light. Without precise timing, it is impossible to have high precision when comparing epochs from different oppositions.

\section{ACKNOWLEDGMENT}

The asteroid program at the University of Arizona is supported by the National Aeronautics and Space Administration.

\section{REFERENCES $^{2}$}

Ahmad, I. I. 1954, Photometric Studies of Asteroids. IV. The Light-Curves of Ceres, Hebe, Flora, and Kalliope. Astrophys. J. 120, 551-559. [1]

Beyer, M. 1953, Der Lichtwechsel und Die Lage der Rotationsachse der Planeten 433 Eros während der Opposition 1951-52. Astron. Nachr. 281, 121-130. [2]

${ }^{2}$ The numbers in brackets are as used in table $I$. 
Calder, W. A. 1935, Photoelectric Photometry of Asteroids. Harvard Bull., no. 904, pp. 11-18.

Chang, Y. C., and Chang, C. S. 1962, Photometric Investigations of Seven Variable Asteroids. Acta Astron. Sinica 10, 101-110. [3]

Chang, Y. C., and Chang, C. S. 1963, Photometric Investigations of Variable Asteroids. II. Acta Astron. Sinica 11, 139-148. [4]

Dunlap, J. L., and Gehrels, T. 1969, Minor Planets. III. Lightcurves of a Trojan Asteroid. Astron. J. 74, 796-803. [5]

Dunlap, J. L., and Gehrels, T. 1971, Minor Planets and Related Objects. VIII. Astron. J. 76, in press. [6]

Fichera, E. 1958, Astron. Nachr. 284, 65-70.

Gehrels, T. 1956, Photometric Studies of Asteroids. V. The Light-Curve and Phase Function of 20 Massalia. Astrophys. J. 123, 331-338. [7]

Gehrels, T. 1957, Photometric Studies of Asteroids. VI. Astrophys. J. 125, 550-570.

Gehrels, T. 1967a, Minor Planets. I. The Rotation of Vesta. Astron. J. 72, 929-938. [8]

Gehrels, T. 1967b, Minor Planets. II. Photographic Magnitudes. Astron. J. 72, 1288-1291.

Gehrels, T. 1970, Photometry of Asteroids. Surfaces and Interiors of Planets and Satellites (ed., Dollfus), pp. 317-375. Academic Press, Inc. London.

Gehrels, T., and Owings, D. 1962, Photometric Studies of Asteroids. IX. Additional Light-Curves. Astrophys. J. 135, 906-924. [9]

Gehrels, T., Roemer, E., Taylor, R. C., and Zellner, B. H. 1970, Minor Planets and Related Objects. IV. Asteroid (1566) Icarus. Astron. J. 75, 186-195. [10]

Giclas, H. L. 1951, Direct Photoelectric Photometry. The Project for the Study of Planetary A tmospheres. Lowell Observ. Rept. 9, pp. 33-72. [11]

Groeneveld, I., and Kuiper, G. P. 1954a, Photometric Studies of Asteroids. I. Astrophys. J. 120, 200-220. [12]

Groeneveld, I., and Kuiper, G. P. 1954b, Photometric Studies of Asteroids. II. Astrophys. J. 120, 529-546. [13]

Haupt, H. 1958, Photoelektrisch-photometrische Studie an Vesta. Mitt. Sonnobs. Kanzelhöhe 14, 172-173. [14]

Hertzsprung, E. 1928, On the Character of the Variation of SX Aurigae. Bull. A stron. Inst. Neth. 4, 178-180.

Hertzsprung, E. 1941, Photographic Estimates of 25 Southern Variable Stars. Bull. Astron. Inst. Neth. 9, 203-215.

Houten-Groeneveld, I. van, and Houten, C. J. van. 1958, Photometric Studies of Asteroids. VII. Astrophys. J. 127, 253-273. [15]

Houten-Groeneveld, I. van, and Houten, C. J. van. 1972, in preparation. [16]

Johnson, H. L. 1963, Photometric Systems. Basic Astronomical Data (ed., A. K. Strand), vol. 3, pp. 204-224. Univ. of Chicago Press. Chicago.

Kuiper, G. P., Fujita, Y., Gehrels, T., Groeneveld, I., Kent, J., Van Biesbroeck, G., and Houten, C. J. van. 1958, Survey of Asteroids. Astrophys. J. Suppl. Ser. 3, 289-427. [17]

Miner, E., and Young, J. 1969, Photometric Determination of the Rotation Period of 1566 I carus. Icarus 10, 436-440. [18]

Miner, E., and Young, J. 1971, Photometric Observations of 1620 Geographos. Icarus, in press. [19]

Müller, G. 1897, Die Photometrie der Gestirne. Engelman. Leipzig.

Sather, R. E., and Taylor, R. C. 1972, Minor Planets and Related Objects. IX. Astron J. 77 , in preparation. [20]

Shatzel, A. V. 1954, Photometric Studies of Asteroids. III. The Light-Curve of 44 Nysa. Astrophys. J. 120, 547-550. [21]

Stephenson, C. B. 1951, The Light-Curve and the Color of Vesta. Astrophys. J. 114, 500-504. [22] 
Stobbe, J. 1940, Der Lichtwechsel des Eros. Astron. Nachr. 270, 1-24.

Taylor, R. C., and Gehrels, T. 1972, Minor Planets and Related Objects. IX. Astron. J. 77, in preparation. [23]

Taylor, R. C., Gehrels, T., and Silvester, A. B. 1971, Minor Planets and Related Objects. VI. Asteroid (110) Lydia. A stron. J. 76, 141-146. [24]

Tempesti, P., and Burchi, R. 1969, A Photometric Research on the Minor Planet 12 Victoria. Mem. Soc. Astron. Ital. Nuova Ser. 40, 415-432. [25]

Univ. of Arizona. 1972, Data to be published by group working with T. Gehrels. [26]

Veverka, J. 1971, Photopolarimetric Observations of the Minor Planet Flora. Icarus, in press. [27]

Veverka, J., and Liller, W. 1969, Observations of Icarus: 1968. Icarus 10, 441-444. [28]

Wood, H. J., and Kuiper, G. P. 1963, Photometric Studies of Asteroids. X. Astrophys. J. 137, 1279-1285. [29] 